\title{
Validação da determinação de $\beta$-hidroxibutirato por meio de glicosímetro portátil em ovelhas para diagnóstico da toxemia da prenhez
}

\author{
Validation of the determination of $\beta$-hydroxybutyrate on dipstick using a portable glucometer in \\ sheep of Dorper and White Dorper \\ Validación de la determinación de $\beta$-hidroxibutirato utilizando un glucómetro portátil en ovejas para \\ el diagnóstico de toxemia gestacional
}

Fernanda Adami Ribeiro ORCID: https://orcid.org/0000-0003-3834-6739 Veterinária Autônoma, Brasil E-mail: fernanda.adami.vet@gmail.com

Ítalo Câmara de Almeida ORCID: https://orcid.org/0000-0002-3349-1796 Universidade Federal do Espírito Santo, Brasil E-mail: almeidaicvet@gmail.com

Tarcísio Ávila dos Santos ORCID: https://orcid.org/0000-0001-6110-7176 Veterinário Autônomo, Brasil

E-mail: tarcisioavila.vet@gmail.com

Leonardo de Bruyn Denadai ORCID: https://orcid.org/0000-0001-6532-205X Veterinário autônomo, Brasil E-mail: leonardodenadaivet@gmail.com

Sayanne Luns Hatum de Almeida ORCID: https://orcid.org/0000-0003-4086-2775

Universidade Federal do Mato Grosso, Brasil E-mail: sayhatum@gmail.com Igor José Faria Silva

ORCID: https://orcid.org/0000-0001-5142-0644 Veterinário Autônomo, Brasil E-mail: igor.fh@hotmail.com Yuri Barbosa Guerson ORCID: https://orcid.org/0000-0001-7796-0921 Veterinário Autônomo, Brasil E-mail: yuriguerson@hotmail.com

Ana Paula Madureira

ORCID: https://orcid.org/0000-0002-6614-7940 Universidade Federal de São João Del Rei, Brasil E-mail: ana_paulamad@hotmail.com

Heberth de Paula

ORCID: https://orcid.org/0000-0001-6197-4165

Universidade Federal do Espírito Santo, Brasil

E-mail: heberth.paula@ufes.br

Jeanne Broch Siqueira

ORCID: https://orcid.org/0000-0002-1903-7554 Universidade Federal dos Vales do Jequitinhonha e Mucuri, Brasil

E-mail: jbsiqueira@@hotmail.com

Graziela Barioni

ORCID: https://orcid.org/0000-0002-2841-6863

Universidade Federal do Espírito Santo, Brasil E-mail: grazibari@gmail.com

\begin{abstract}
Resumo
Devido ao crescimento da ovinocultura e o aumento da demanda em relação aos seus produtos, há a necessidade de se investir na sanidade desses animais. Com isso, torna-se imprescindível a utilização de meios diagnósticos para afecções metabólicas, principalmente a toxemia da prenhez. Esta pesquisa tem como objetivo validar a técnica diagnóstica em fita reagente para $\beta$-hidroxibutirato (BHB) em glicosímetro portátil humano em ovelhas da raça Dorper e White Dorper. Foram utilizadas 111 ovelhas hígidas, 79 da raça Dorper e 32 White Dorper, em diferentes fases produtivas, sendo elas
\end{abstract}


vazias $(n=44)$, gestantes $(n=37)$ e recém-paridas $(n=30)$. A coleta de sangue foi realizada por punção da veia jugular, com sistema de coleta a vácuo, em tubo sem anticoagulante. Instantaneamente, foi realizada a determinação do BHB pelo método da fita reagente utilizando-se o glicosímetro portátil e a determinação do BHB no soro foi realizada em analisador bioquímico automático. A análise estatística foi realizada, mediante o teste de Kolmogorov-Smirnov ( $\mathrm{p}<0,05)$, verificou-se a distribuição não paramétrica dos dados, sendo eles arranjados de maneira pareada, ao qual o mesmo indivíduo era analisado pelas duas técnicas, foi escolhido o teste de McNemar para a verificação da hipótese de diferença entre os testes. Além disso, foi realizado o cálculo do coeficiente Kappa (IC 95\%) para verificar a reprodutibilidade dos testes, associado aos cálculos de sensibilidade e especificidade, considerando o teste laboratorial como padrão ouro. Não houve diferença estatística significativa entre os resultados da fita reagente para corpos cetônicos e da análise bioquímica em laboratório, considerando o total de 111 animais. Nesta análise, a estatística resultou em um índice Kappa de $85 \%$, com sensibilidade da fita reagente de $93 \%$ e especificidade de $96 \%$. Na classificação recém-parida, o teste McNemar ( $\mathrm{p}=0,3173$ ) com IC 95\% demonstrou haver um coeficiente Kappa 80,6\%, com $93 \%$ de sensibilidade e $87 \%$ de especificidade. As ovelhas recém-paridas, nesta categoria de grupos, possuem resultados pareados e, desta forma apresentou um coeficiente Kappa de 80,6\%, com 93\% de sensibilidade e $87 \%$ de especificidade. $O$ teste da fita reagente para $\beta$-hidroxibutirato em glicosímetro portátil humano foi considerado excelente e confiável, podendo ser utilizado como recurso diagnóstico em ovinos.

Palavras-chave: Corpos cetônicos; Ovinos; Diagnóstico.

\begin{abstract}
Due to the growth of sheep breeding and the increase in demand for their products, there is the need to invest in the health of these animals. Thus, it becomes essential to use diagnostic tools for metabolic diseases like toxemia of pregnancy. This research aims to validate the diagnostic technique in reagent strip for $\beta$-hydroxybutyrate (BHB) in human portable glucose in sheep of Dorper and White Dorper. 111 otherwise healthy sheep, 79 of 32 Dorper and White Dorper were used in different production phases, which were empty $(n=44)$, patients $(n=37)$ and recently calved $(n=30)$. Blood collection was performed by puncturing the jugular vein, with vacuum collection system, without anticoagulant tube. Instantly, was performed to determine the BHB test strip by the method of using the portable glucose meter. The determination of BHB in serum was performed in an automatic biochemical analyzer. Analysis, using the KolmogorovSmirnov test $(\mathrm{p}<0.05)$, there was a non-parametric distribution of the data, they are arranged in a paired manner, to which the same individual was examined by two techniques, the test was chosen McNemar to verify the difference between hypothesis testing. In addition, we performed the calculation of Kappa coefficient (95\%) to check the reproducibility of the tests, combined with sensitivity and specificity calculations, considering the laboratory test as the gold standard. There was no significant difference between the results of dipstick to ketone bodies and biochemistry laboratory analysis, considering the total of 111 animals. In this analysis, the statistical resulted in a Kappa Index of $85 \%$, with a sensitivity of the reagent strip $93 \%$ and specificity of $96 \%$. In animals divided into different breeding groups, because there is no disagreement results, it is considered the Kappa index $100 \%$ and thus no possibility of calculating the sensitivity and specificity. This was only possible in newly calved classification of animals. In this category, the McNemar test ( $\mathrm{p}=0.3173$ ) with 95\% demonstrated a Kappa coefficient $80.6 \%$, with 93\% sensitivity and $87 \%$ specificity. The recently calved sheep in this category groups have paired results and thus presented a Kappa coefficient of $80.6 \%$, with $93 \%$ sensitivity and $87 \%$ specificity. The test reagent strip for $\beta$-hydroxybutyrate in human portable blood glucose meter is considered excellent and reliable and can be used as a diagnostic tool in sheep.
\end{abstract}

Keywords: Ketone bodies; Sheep; Diagnosis.

\title{
Resumen
}

Debido al crecimiento de la cría de ovejas y la mayor demanda de sus productos, existe la necesidad de invertir en la salud de estos animales. Por tanto, es fundamental utilizar medios de diagnóstico para los trastornos metabólicos, especialmente la toxemia del embarazo. Esta investigación tiene como objetivo validar la técnica de diagnóstico utilizando una tira reactiva para $\beta$-hidroxibutirato (BHB) en un glucómetro humano portátil en ovejas Dorper y White Dorper. Un total de 111 ovejas sanas, 79 Dorper y 32 White Dorper, fueron utilizadas en diferentes fases de producción, las cuales estuvieron vacías $(n=44)$, preñadas $(n=37)$ y recién paridas $(n=30)$. La extracción de sangre se realizó mediante punción de la vena yugular, con sistema de recolección al vacío, en un tubo sin anticoagulante. Instantáneamente, la determinación de BHB se realizó mediante el método de tira reactiva utilizando un glucómetro portátil y la determinación de BHB en suero se realizó en un analizador bioquímico automático. El análisis estadístico se realizó mediante la prueba de Kolmogorov-Smirnov ( $\mathrm{p}<0.05$ ), se verificó la distribución no paramétrica de los datos, estando ordenados de forma pareada, en la que se analizó el mismo individuo por las dos técnicas. Se eligió la prueba de McNemar para verificar la hipótesis de diferencia entre las pruebas. Además, se realizó el cálculo del coeficiente Kappa (IC 95\%) para verificar la reproducibilidad de las pruebas, asociado a los cálculos de sensibilidad y especificidad, considerando la prueba de laboratorio como estándar de oro. No hubo diferencia estadísticamente significativa entre los resultados de la tira reactiva para cuerpos cetónicos y el análisis bioquímico de laboratorio, considerando un total de 111 animales. En este análisis, las estadísticas dieron como resultado un índice Kappa del 85\%, con una sensibilidad de la tira reactiva del $93 \%$ y una especificidad del $96 \%$. En la clasificación de los recién nacidos, la prueba de McNemar ( $\mathrm{p}=0,3173$ ) con IC del 95\% mostró un coeficiente Kappa del 80,6\%, con una sensibilidad del 93\% y una especificidad 
del $87 \%$. Las ovejas recién paridas, en esta categoría de grupos, tienen resultados apareados y, por lo tanto, presentaron un coeficiente Kappa del $80,6 \%$, con una sensibilidad del $93 \%$ y una especificidad del $87 \%$. La prueba de tiras reactivas para $\beta$-hidroxibutirato en un glucómetro humano portátil se consideró excelente y confiable, y puede usarse como recurso de diagnóstico en ovejas.

Palabras clave: Cuerpos cetónicos; Ovino; Diagnóstico.

\section{Introdução}

Em ovelhas, a gestação pode ser considerada o período mais delicado de sua vida, onde há o aumento de suas necessidades nutricionais, principalmente em animais que apresentem gestações múltiplas e que se encontrem em terço final da gestação. Isso se deve a diversos fatores, entre eles o aumento da demanda energética, a mobilização de reservas corporais e a diminuição da matéria seca consumida em consequência da compressão do rúmem pelo útero gestante que se encontra aumentado, o que acarreta em balanço energético negativo (BEM) (Santos et al. 2011, Nasciutti et al. 2012, Oliveira et al. 2014, Araújo et al., 2020).

Essa diminuição do consumo alimentar das fêmeas juntamente com o aumento das demandas energéticas, acarreta em mobilização de outros tipos de reservas energéticas corporais, sendo a principal, a reserva de lipídeos (Silva et al. 2011, Nasciutti, Bofá, Borges 2012). Essa mobilização eleva o nível de ácidos graxos livres plasmáticos e corpos cetônicos resultando num quadro de cetose, conhecido nos pequenos ruminantes como toxemia da prenhez (Crilly et al., 2021).

O uso de análises sanguíneas como ferramenta de diagnóstico tem se mostrado eficiente para a detecção de enfermidades de caráter metabólico que além de serem de difícil percepção, diminuem constantemente a produção animal, reduzindo a rentabilidade do setor agropecuário. Assim, métodos diagnósticos que permitam avaliar a sanidade desses animais, e que sejam de baixo custo, devem ser empregados (Hernández et al., 2020).

A determinação de corpos cetônicos pode ser realizada no sangue, urina e leite, sendo o padrão mais confiável o diagnóstico de Beta-hidroxibutirato (BHB) no soro (Voyvoda, Erdogan 2010). Karagiannis et al. (2014) afirmaram que o valor de BHB pode ser considerado um bom preditor do estado da saúde no periparto. Ele também reflete o BEM, e por isso a determinação do BHB pode predizer o estado energético durante essa fase reprodutiva. Duffield (2000) destaca que na literatura existem relatos de vários testes capazes de dosar o BHB: Rothera, bioquímico laboratorial, tira reagente em glicosímetro portátil e ainda o Ensaio de Imunoabsorbância Ligado a Enzima (ELISA), utilizado somente para fins de pesquisa (Eurodiagnóstico 2014).

O teste bioquímico laboratorial é considerado o padrão ouro para a determinação quantitativa de BHB, porém, este método tem como desvantagem ser mais oneroso e que demanda tempo para o envio das amostras e realização do exame (Doré et al. 2013). Como alternativa Nasciutti et al. (2012) e Iwersen et al. (2013) destacaram o uso de dispositivos portáteis, utilizados no controle da diabetes em humanos, para a deteç̧ão desses corpos cetônicos em vacas e ovelhas.

Este trabalho tem como objetivo validar a técnica de determinação de BHB em glicosímetro portátil humano, por meio de fita reagente, em ovelhas da raça Dorper e White Dorper.

\section{Metodologia}

O trabalho relativo ao projeto de pesquisa intitulado "Perfil Metabólico em Ovinos" foi aprovado pela Comissão de Ética do Uso de Animais (CEUA), da Universidade Federal do Espírito Santo (UFES) atendendo as normas éticas, conforme estabelecidas na legislação vigente e no regime interno da CEUA-UFES, sob o protocolo nº 019/2014.

Foram utilizadas 111 ovelhas adultas, 79 da raça Dorper e 32 da raça White Dorper, nos seguintes períodos reprodutivos: 37 gestantes, 30 recém paridas e 44 vazias. O manejo sanitário era realizado com vacinação anual contra raiva e clostridioses e 
a vermifugação realizada com base no resultado da contagem de ovos por grama de fezes (OPG), sendo o princípio ativo escolhido pela eficácia.

O manejo nutricional das ovelhas vazias e paridas era semiextensivo. Durante o dia ficavam soltas em pasto de Brachiaria spp e ao entardecer confinadas. Durante o confinamento as ovelhas vazias recebiam apenas sal mineralizado próprio para espécie; as recém paridas além do sal mineral, ganhavam capim picado ou silagem de milho no cocho e $1 \%$ do peso vivo de suplementação com concentrado, podendo chegar a $2 \%$ dependendo da qualidade da pastagem. No pré-parto as ovelhas ficavam em sistema de confinamento e recebiam capim picado ou silagem de milho à vontade no cocho, concentrado na proporção de $1 \%$ do peso vivo por dia e sal mineral à vontade.

As ovelhas foram previamente selecionadas por meio de exame de inspeção, avaliação de mucosas pelo método Famacha $\odot$ (Chagas et al., 2007), sendo utilizados apenas os animais considerados hígidos. A coleta de sangue foi realizada por punção de veia jugular, com sistema de coleta a vácuo, em tubo sem anticoagulante. Os tubos com as amostras de sangue foram acondicionados em isopor com gelo reciclável, sem ultrapassar quatro horas em seu interior, até serem centrifugadas a $3000 \mathrm{rpm}$ por 10 minutos para a separação do soro. O soro foi separado e aliquotado em tubos de polietileno de $1,5 \mathrm{~mL}$ previamente identificados e armazenados a $-20^{\circ} \mathrm{C}$ até o momento da realização do exame bioquímico.

A determinação do $\beta$-hidroxibutirato em fita reagente, foi realizada, imediatamente após a coleta, por meio de uma gota de sangue total, colocada sobre área específica da fita (Fita FreeStyle Optium $\beta$-ketone, Abbott) inserida em um glicosímetro portátil humano (Optium Xceed, Abbott), que determinouo BHB em mmol/L no sangue. A determinação bioquímica de BHB foi realizada seguindo as instruções do kit comercial (Ranbut, Randox ${ }^{\circledR}$ ), em analisador bioquímico automático (Mindray BS 120, Bioclin).

Por apresentarem uma distribuição não paramétrica (verificada mediante teste de Kolmogorov-Smirnov) $(\mathrm{p}<0,05)$ e serem arranjados de maneira pareada, onde o mesmo indivíduo foi avaliado pelas duas técnicas, os resultados foram avaliados mediante teste de McNemar no intuito de verificar a hipótese de diferença entre os testes. Além disso, foi realizado o cálculo do coeficiente Kappa (IC 95\%) para verificar a reprodutibilidade dos testes e os cálculos dos valores de sensibilidade e especificidade considerando-se o teste em laboratório como padrão ouro. Para cálculo da sensibilidade e especificidade foram considerados animais positivos, ou seja, que apresentaram toxemia da prenhez, aqueles animais que apresentaram valores de BHB superiores a $0,6 \mathrm{mmol} / \mathrm{L}$.

\section{Resultados}

Não houve diferença estatística significativa entre os resultados da fita reagente para corpos cetônicos e da análise bioquímica em laboratório, considerando o total de 111 animais (Tabela 1). Nesta análise, o teste McNemar ( $\mathrm{p}=0,3173$ ) com IC 95\% resultou em um índice Kappa entre os testes de fita reagente e laboratorial de 85\%, com sensibilidade da fita reagente de $93 \%$ e especificidade de $96 \%$. No gráfico de dispersão (Figura 1), está demonstrada a proximidade dos valores dos exames de fita reagente com glicosímetro portátil e exame bioquímico laboratorial nas 111 ovelhas avaliadas. 
Tabela 1 - Valores de média e desvio padrão de $\beta$-hidroxibutirato (BHB) determinados pelos métodos de fita reagente em glicosímetro portátil humano (BHB Fita) e análise bioquímica laboratorial (BHB Laboratorial), em 111 ovelhas das raças Dorper e White Dorper em diferentes fases produtivas e em cada fase produtiva.

\begin{tabular}{ccc}
\hline Métodos $(\mathbf{m m o l} / \mathbf{L})$ & Média $(\mathbf{m m o l} / \mathbf{L})$ & Desvio Padrão \\
\hline BHB Fita & 0,4 & 0,2 \\
\hline BHB Laboratorial & 0,4 & 0,2
\end{tabular}

\begin{tabular}{ccc}
\hline Fases Produtivas & BHB Fita $(\mathbf{m m o l} / \mathbf{L})$ & BHB Laboratorial $(\mathbf{m m o l} / \mathbf{L})$ \\
\hline Vazias $(\mathbf{n}=\mathbf{4 4})$ & $0,3 \pm 0,1$ & $0,3 \pm 0,1$ \\
\hline Gestantes $(\mathbf{n}=\mathbf{3 7})$ & $0,3 \pm 0,1$ & $0,3 \pm 0,1$ \\
\hline Recém Paridas $(\mathbf{n}=\mathbf{3 0})$ & $0,6 \pm 0,4$ & $0,6 \pm 0,3$ \\
\hline
\end{tabular}

Fonte: Autores.

Os animais divididos em grupos reprodutivos vazias e gestantes não alcançaram valores de BHB superiores ao nível de corte $(0,6 \mathrm{mmol} / \mathrm{L})$, não sendo possível realizar o cálculo para determinação da sensibilidade e a especificidade do método com a fita reagente (Tabela 1). No entanto para o grupo de animais classificados como recém paridas, esse cálculo foi possível e o teste McNemar ( $p=0,3173$ ) com IC 95\% demonstrou haver um coeficiente Kappa 80,6\%, com 93\% de sensibilidade e 87\% de especificidade. Além disso foi possível observar que tanto a sensibilidade quanto a especificidade apresentaram valores excelentes a nível de exatidão para o teste em fita reagente.

Figura 1 - Gráfico de dispersão demonstrando valores obtidos para $\beta$-hidroxibutirato em tira reagente em glicosímetro portátil (BHB Fita) e com o exame bioquímico laboratorial (BHB Bioq), das 111 ovelhas avaliadas.

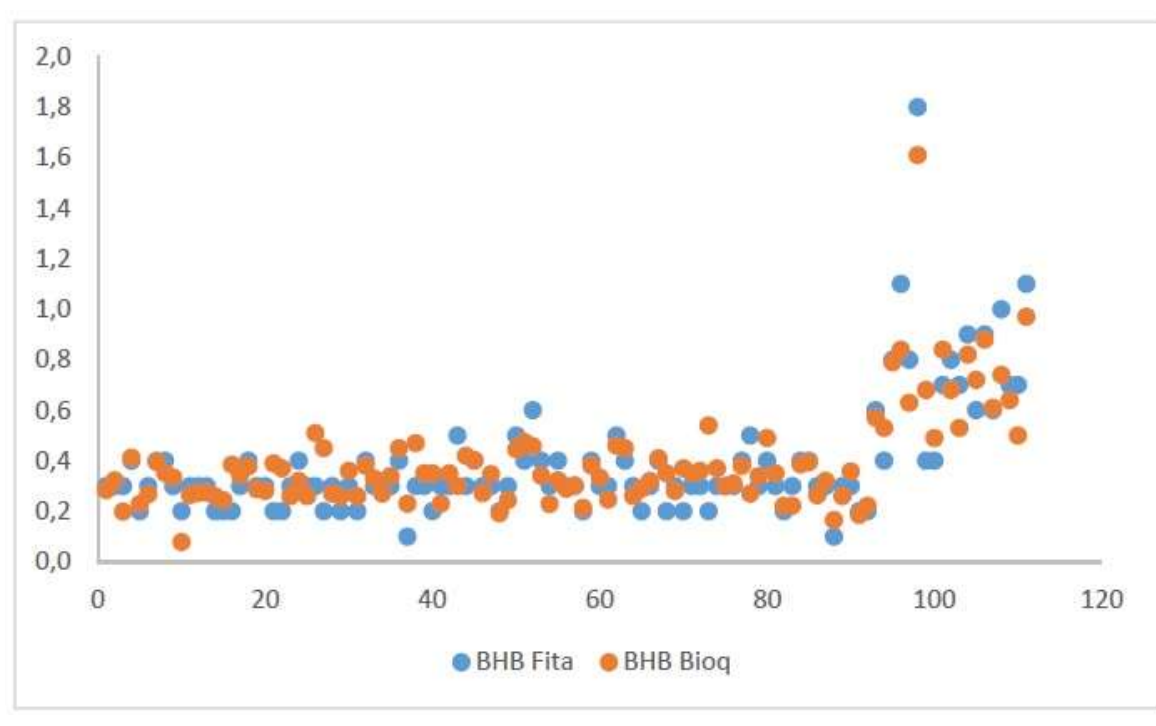

Fonte: Autores.

Com relação aos valores de BHB nas diferentes fases produtivas não foi observada diferença significativa entre os grupos, e todos se encontraram dentro dos limites de referência de 0,6mmol/L (Contreras, Wittwer, Böhmwald 2000). Entretanto, notou-se que os níveis de BHB foram mais elevados em ovelhas recém paridas. 


\section{Discussão}

Os coeficientes Kappa encontrados nesse experimento, $85 \%$ e 80,6\%, podem ser considerados de boa a ótima concordância, assim como descrito por Pereira (1995). Esse autor afirma ainda que a validade de um teste diagnóstico parte do princípio que o resultado pode ser aceito como expressão da verdade ao observar-se a sensibilidade e especificidade. A sensibilidade determina a capacidade máxima que o teste tem para diagnosticar indivíduos verdadeiramente positivos para doença, enquanto a especificidade define a capacidade que o teste possui em diagnosticar corretamente os indivíduos sadios.

Panousis et al. (2011), também observou resultados semelhantes em experimento similar com vacas, obtendo uma sensibilidade de $90 \%$ e especificidade de 97\%. Em 2012, Panousis et al. com o intuito de obter os valores de BHB em ovinos em período seco e em lactação, utilizou o aparelho Precision Xceed® e observou uma sensibilidade de 98,6\% e especificidade de $98,2 \%$. Essas medidas foram semelhantes às obtidas pelo teste laboratorial. No presente estudo, as ovelhas recém paridas, também apresentaram alta sensibilidade ao teste de fita reagente, assim como Panousis et al. (2012), porém a especificidade foi menor que a apresentada por esse autor. A maior especificidade encontrado por Panousis et al. (2012), se deu provavelmente pelo maior número de animais analisados.

A determinação dos valores de BHB pelo glicosímetro portátil e a fita reagente, apresentaram concordância ótima com a determinação bioquímica de BHB, o que também foi demonstrado por Pichler et al. (2014) em seu experimento com ovelhas.

Voyvoda e Erdogan (2010), Panousis et al. (2012) e Pichler et Al. (2014) não constataram diferença significativa entre o teste laboratorial com o de fita reagente para corpos cetônicos, concordando com os resultados obtidos no presente trabalho. Esses resultados comprovam a afirmação desses autores, de que a fita reagente é um teste benéfico para o monitoramento de animais com risco de cetonemia, e no caso de ovelhas, toxemia gravídica.

A utilização do glicosímetro portátil e a fita reagente se mostrou um método rápido, ágil, confiável e eficiente na determinação de valores de BHB, validando a possibilidade de ser utilizado como método de diagnóstico para toxemia da prenhez clínica e subclínica. Segundo Voyvoda e Erdogan (2010) e Panousis et al. (2011), a importância do estudo deste método está na precocidade do diagnóstico de doenças que possam causar queda na produção ou perda de animais no rebanho, o que acarreta em prejuízos econômicos.

Haja vista que o kit bioquímico custa, em média R\$434,38, e possui capacidade para efetuar 200 exames, o custo de cada exame seria de no mínimo $\mathrm{R} \$ 2,17$. As fitas reagentes FreeStyle ${ }^{\circ}$ utilizadas no exame com glicosímetro portátil Option Xceed® em contrapartida custam cerca $\mathrm{R} \$ 2,99$ cada, e o aparelho portátil, em média $\mathrm{R} \$ 50,00$. Constata-se dessa forma, que o custo de cada exame é equivalente, porém é importante atenta-se que para a realização do exame bioquímico custos adicionais devem ser levados em conta, como é o preço dos aparelhos bioquímicos, custos com envio das amostras, e taxa cobrada pelo laboratório. Portanto o método de dosagem de BHB em glicosímetro portátil além de mais rápido é também mais barato como afirmam Voyvoda e Erdogan (2010) e Doré et al. (2013).

\section{Conclusão}

A dosagem de BHB por meio da fita reagente em glicosímetro portátil se mostrou um método apropriado, confiável e de simples execução, para ovinos, no diagnóstico de alterações de caráter metabólico energético tais como cetose e toxemia da prenhez. Por ser um método rápido e relativamente barato pode auxiliar no trabalho do médico veterinário a campo, facilitar o diagnóstico e contribuir para o tratamento mais precoce e assim evitar prejuízos a produção e saúde do animal.

\section{Referências}

Araújo, C. A. S. C., Minervino, A. H. A., Sousa, R. S., Oliveira, F. L. C., Rodrigues, F. A. M. L., Mori, C. S. \& Ortolani, E. L. (2020). Validation of a handheld $\beta$-hydroxybutyrate acid meter to identify hyperketonaemia in ewes. Biochemistry, Biophusics and Molecular Biology, 8:8933-8942. 
Chagas, A. C. S., Oliveira, M. C. S., Carvalho, C. O. \& Molento, M. B. (2007). Método Famacha@: Um recurso para o controle de verminose em ovinos. Circular técnica 52, 8p. Embrapa Pecuária Sudeste.

Crilly, J. P., Phythian, C. \& Evans, M. (2021). Advances in managing pregnancy toxaemia in sheep. In Practice, 43:79-94.

Contreras, P., Wittwer, F. \& Böhmwald H. (2000). Uso dos perfis metabólicos no monitoramento nutricional de ovinos. In: González F.H.D., Barcellos J.O., Ospina H., Ribeiro L.A.O. Perfil metabólico em ruminantes: seu uso em nutrição e doenças nutricionais. Gráfica da Universidade Federal do Rio Grande do Sul, Porto Alegre. 75-88.

Doré, V., Dubuc, J., Bélanger A. M. \& Buczinski S. (2013). Short communication: Evaluation of the accuracy of na eletronic on-farm test to quantify blood $\beta$ hydroxybutyrate concentration in dairy goats. Journal Dairy Sciencex, 96(7), 4505-4507.

Duffield, T. (2000). Subclinical ketosis in lactating dairy cattle. Veterinary Clinics of North America. Food Animal Practice, 16:231-253.

Eurodiagnóstico (2015). Beta-Hydroxybutyrate (beta-HB) Assay Kit.

Hernández, J., Benedito, J. L., \& Castillo, C. (2020). Relevance of the study of metabolic profiles in sheep and goat flock. Spanish Journal of Agricultural Research, 18:1-14.

Iwersen, M., Kklein-Jobstl, D., Pichler, M., Roland, L., Fildlschuster, B., Scchwendenwein, I. \& Drillich, M. (2013). Comparason of 2 electronic cowside tests to detect subclinical ketosis in dairy cows and the influence of the temperature and type of blood sample on the tests results. American Dairy Science Association, 96(12), 7719-7730.

Karagiannis, I., Panousis, N., Kiossis, E., Tsakmakidis, I., Lafi, S., Arsenos, G., Boscos, S.C. \& Brozos, C. (2014). Associations of pre-lambing body condition score and sérum $\beta$-hydroxybutyric acid and non-esterified fatty acids concentration with peroparturient health of chios dairy ewes. Small Ruminant Research, 120(1), 164-173.

Nasciutti, N. R., Tsuruta, S. A., Oliveira, R. S. B. R., Bisinoto, M., Headley, S. A., Mundim, A. V., Noleto, P. G. \& Sault, J. P. E. (2012). Perfil metabólico em ovelha Santa Inês, com baixo escore de condição corporal, no periparto. Boletim de Indústria Animal, 69(2), 137-145.

Oliveira, R. P. M., Maduro, A. H. P., Oliveira, F. F. \& Lima, E. S. (2014). Perfil metabólico de ovelhas Santa Inês em diferentes fases de gestação criadas em sistema semi-intensivo no estado do Amazonas. Ciência Animal Brasileira, 15(1), 81-86.

Panousis, N., Kritsepi, M., Karagiannis, I., Kalaitzakis, E., Lafi, S. \& Brozos, C. (2011). Evaluation of precision Xceed for on-site monitoring of blood $\beta$ hydroxybutyric acid and glucose in dairy cows. Jounal of the Ellenic Veterinary Medical Society, 62(2), 109-117.

Panousis, N., Brozos, C., Karagiannis, I., Giadinis, N. D., Lafi, S. \& Kritsepi-Konstantinou, M. (2012). Evaluation of Precision Xceed® meter for on-site monitoring of blood $\beta$-hydroxybutyric acid and glucse concentration in dairy sheep. Research in Veterinary Science, 93, 435-439.

Pereira M. G. (1995). Epidemiologia: teoria e prática. Rio de Janeiro. 363p. Editora Guanabara Koogan.

Pichler, M., Damberger, A., Arnholdt, T., Schwendenwein, J., Gasteiner, J., Drillich, M. \& Iwersen, M. (2014). Evaluation of 2 eletronic handheld devices of diagnosis of cetonemia and glycemia and dairy goats. Journal Dairy Science, 97(12), 7538-7546.

Santos, F. C. O., Mendonça, C. L., Silva-Filho, A. P., Carvalho, C. C., Soares, P. C. \& Afonso J. A. B. (2011). Indicadores bioquímicos e hormonais de casos naturais de toxemia da prenhez em ovelhas. Pesquisa Veterinária Brasileira, 31(11), 974-980.

Silva, N. C. D., Bonfá, H. C. \& Borges, I. (2011). Cetose em Pequenos Ruminantes. Revista Eletrônica Nutritime, 8(6), 1647-1657.

Simões, P. B. A., Bexiga, R., Lamas, L. P., \& Lima, M. S. (2020). Pregnancy Toxaemia in Small Ruminants. In Advances in Animal Health. Medicine and Production, pp. 541-556. Springer, Cham.

Voyvoda, H. \& Erdogan H. (2010). Use of hand-held meter for detecting subclinical ketosis in dairy cows. Research in Veterinary Science, 89(3), 344-351. 